\title{
Modeling the Social and Epidemiological Causes of Hearing Loss
}

\section{Modelando las Causas Sociales y Epidemiológicas de la Pérdida de Audición}

\author{
F. Kouilily', F. Z. Aboulkhouatem', N. Yousfi', N. Achtaich', M. El Khasmi \\ 'Department of Mathematics and Computer Science, Faculty of Science Ben M'sik Hassan II University \\ 2Department of Biology, Faculty of science Ben M'sik Hassan II University
}

\section{ABSTRACT}

Hearing loss result from genetic causes, complications at birth, certain infectious diseases, chronic ear infections, noise exposure, demographic characteristics (age, sex, race, education, and study site) and cardiovascular factors (smoking status, hypertension, diabetes and stroke). In this study, we propose a new mathematical model formulated by ordinary differential equations (ODEs) that takes into account the some causes of hearing loss. The analysis of the model is investigated. In addition, numerical simulations are presented in order to validate our theoretical results.

KEYWORDS: viral infection; ordinary differential equations; stability 


\section{RESUMEN}

La pérdida de audición se debe a causas genéticas, complicaciones en el nacimiento, enfermedades infecciosas, otitis crónica, exposición al ruido, características demográficas (edad, sexo, raza, educación y sitio de estudio) y factores cardiovasculares (estado de fumar, hipertensión, diabetes y accidente vascular cerebral). En este estudio, proponemos un nuevo modelo matemático formulado por ecuaciones diferenciales ordinarias (ODE) que toma en cuenta las causas de la pérdida de la audición. El análisis del modelo se estudia. Además, se presentan simulaciones numéricas para validar nuestros resultados teóricos.

PALABRAS CLAVE: infección viral; ecuaciones diferenciales ordinarias; estabilidad

\section{Correspondencia}

DESTINATARIO: Kouilily Fatiha

INSTITUCIÓN: Department of Mathematics and

Computer Science, Faculty of Science Ben M'sik

Hassan II University

DIRECCIÓN: P. O. Box 7955, Sidi Othman,

Casablanca, Morocco

CORREOELECTRÓNICO: kouililyfatiha@gmail.com

\section{Fecha de recepción:}

21 de enero de 2018

Fecha de aceptación:

3 de julio de 2018 


\section{INTRODUCTION}

Hearing loss may result from genetic causes, complications at birth, certain infectious diseases, chronic ear infections, the use of particular drugs, exposure to excessive noise and aging, which is represent a major global health problem. According to the World Health Organization (WHO), about 360 million people worldwide have disabling hearing loss, and 32 million of these are children ${ }^{[1]}$. There are many infections and contagious diseases related to hearing loss such as mumps. Mumps is an enveloped, single-stranded RNA virus belonging to the family paramyxoviridae and causes an acute infectious disease mainly in children and young adults ${ }^{[2]}$. Mumps is transmitted through infected respiratory secretions and is highly contagious ${ }^{[3]}$. The most common clinical manifestations of infection include a u-like illness and bilateral swelling of the parotid glands. Mumps infection occasionally induces the potential for complications such as pancreatitis, orchitis, ophoritis, aseptic meningitis, encephalitis and sensorineural hearing loss. Hearing loss due to mumps is thought to be unilateral and profound with rapid onset ${ }^{[4]}$.

On the other hand, one of the most social factor of hearing loss is noise exposure (eg, through personal music players). This is a major cause of hearing loss worldwide ${ }^{[5]}$. In addition, the characteristic pathological feature of noise-induced hearing loss is the loss of auditory sensory cells in the cochlea. Because these hair cells cannot regenerate in mammals, no remission can occur, prevention of noise-induced hearing loss is the only option to preserve hearing ${ }^{[6]}$.

Abnormalities or damage in the structure inside the inner ear can result by many diseases that can load to the hearing loss. An example of such a disease is the Alport syndrome ${ }^{[7]}$ and Meniere's disease ${ }^{[8]}$. For these raisons, many mathematical models have been proposed to model the function and the dysfunction of the inner ear by using partial differential equation (PDEs) [7, $8,9,10,11]$. The epidemiological models in the beginning has started by Graunt ${ }^{[12]}$ then described by Kermack and Mckendric ${ }^{[3]}$ by considering the total population into three classes namely susceptible (S) individuals, infected (I) individuals and recovered (R) individuals which is known to us as SIR epidemic model ${ }^{[14,15,16]}$. Today, this SIR epidemic model is very important in analysis of many diseases. Referring to the simplest version of the most classical epidemiological model for directly-transmitted infectious diseases ${ }^{[17,18,19,20,21,22]}$, as well as, the social and epidemiological factors of hearing loss existing in the biological studies and in the literature [23, 24, 25], we proposed a new mathematical model by using (ODEs) that describe the time dependence of the dynamics of hearing loss by considering the contagion factor is the mumps virus and the social factor is the exposure to noise. This model is described by the following system:

$$
\begin{aligned}
& \frac{d H}{d t}=\Lambda-\mu H-[\beta L+\varepsilon] H, \\
& \frac{d L}{d t}=[\beta L+\varepsilon] H-(\mu+\gamma) L, \\
& \frac{d R}{d t}=\gamma L-\mu R .
\end{aligned}
$$

The population is divided into three epidemiological classes that are: $\mathrm{H}(\mathrm{t})$ is the number of susceptible individuals at time $\mathrm{t}$ (normal hearing), $\mathrm{L}(\mathrm{t})$ is the number of infected individuals at time $t$ (loss of hearing), and $\mathrm{R}(\mathrm{t})$ is the number of removed individuals at time $\mathrm{t}$ (recovered hearing). Further $\Lambda$ is the recruitment rate of the population, $\mu$ is the natural death rate of the population, $\beta$ is the transmission rate due to social contagion of hearing loss (mumps), $\varepsilon$ is the non-contagion risk of hearing loss due to noise exposure and $\gamma$ is the recovery rate of the infective individuals.

The rest of paper is organized as follows. In the next section, positivity and boundedness of solutions are studied. In Section 3, the basic reproduction number is derived, also the local and the global asymptotic stability of the equilibria are analyzed. The numerical results are given in Section 4. Lastly, we give a conclusion of our results in Section 5. 


\section{Positivity and boundedness of solutions}

In this section, we will establish the positivity and boundedness of solutions of model (1), which proves that our model is well posed.

PROPOSITION 2.1. All solutions starting from non-negative initial conditions exist for all $\mathrm{t}>0$ and remain bounded and non-negative.

PROOF. For the positivity, we show that any solution starting in non-negative orthant, $\mathbb{R}^{3}=\left\{(\mathrm{H}, \mathrm{L}, \mathrm{R}) \in \mathbb{R}^{3}\right.$ : $\mathrm{H} \geq 0, \mathrm{~L} \geq 0, \mathrm{R} \geq 0\}$. In fact, $(\mathrm{H}(\mathrm{t}), \mathrm{L}(\mathrm{t}), \mathrm{R}(\mathrm{t})) \in \mathbb{R}^{3}{ }_{+}$and we have:

$$
\begin{aligned}
& \left.\frac{d H}{d t}\right|_{H=0}=\Lambda \\
& \left.\frac{d L}{d t}\right|_{L=0}=\varepsilon H \\
& \left.\frac{d R}{d t}\right|_{R=0}=\gamma L .
\end{aligned}
$$

This proves the positivity of solutions. Now, we prove that the solutions are bounded. We defined $\mathrm{T}(\mathrm{t})=\mathrm{H}(\mathrm{t})+\mathrm{L}(\mathrm{t})$ By non-negativity of the solution, it follows that;

$$
\begin{gathered}
\frac{d T(t)}{d t}=\Lambda-\mu H-(\mu+\gamma) L \\
\frac{d T(t)}{d t} \leq \Lambda-\mu(H+L)
\end{gathered}
$$

Then

$$
\lim _{t \rightarrow \infty} \sup T(t) \leq \frac{\Lambda}{\mu}+(H(0)+L(0)) e^{-\mu t} \leq \frac{\Lambda}{\mu}
$$

From the last equation (1) and since $L$ is bounded, we deduce that $\mathrm{R}$ is bounded. This completes the proof.

\section{Stability analysis of hearing loss model}

\section{Local stability of endemic equilibrium point for $\varepsilon>0$}

The following theorem presents the existence and uniqueness of endemic equilibrium if $R_{0}>1$.
THEOREM 3.1. System (1) has a unique equilibrium point $E_{\varepsilon}^{*}$, which is locally asymptotically stable.

PROOF. By equalizing to zero the rights members of the system (1), we find one endemic point that exists for above model:

$$
E_{\varepsilon}^{*}=\left(H_{\varepsilon}^{*}, L_{\varepsilon}^{*}, R_{\varepsilon}^{*}\right)
$$

Where

$$
\begin{aligned}
H_{\varepsilon}^{*} & =\frac{2 \Lambda(\mu+\gamma)}{\beta \Lambda+(\varepsilon+\mu)(\gamma+\mu)+\sqrt{\Delta}} \\
L_{\varepsilon}^{*} & =\frac{\beta \Lambda-(\varepsilon+\mu)(\gamma+\mu)+\sqrt{\Delta}}{2 \beta(\mu+\gamma)} \\
R_{\varepsilon}^{*} & =\gamma \frac{\beta \Lambda-(\varepsilon+\mu)(\gamma+\mu)+\sqrt{\Delta}}{2 \mu \beta(\mu+\gamma)}
\end{aligned}
$$

With

$$
\Delta=(\beta \Lambda-(\varepsilon+\mu)(\gamma+\mu))^{2}+4 \beta \varepsilon \Lambda(\gamma+\mu)
$$

The Jacobian matrix of the system (1) is given by:

$$
J=\left(\begin{array}{ccc}
-\mu-(\beta L+\varepsilon) & -\beta H & 0 \\
\beta L+\varepsilon & \beta H-(\gamma+\mu) & 0 \\
0 & \gamma & -\mu
\end{array}\right)
$$

The characteristic equation of the endemic equilibrium point is given by;

$$
(-\mu-\lambda)\left(\lambda^{2}+C_{1} \lambda+C_{2}\right)=0
$$

Note that coefficient

$$
\left\{\begin{array}{c}
C_{1}=\varepsilon+\gamma+\beta L_{\varepsilon}^{*}-\beta H_{\varepsilon}^{*}+2 \mu \\
C_{2}=\varepsilon \gamma+\beta \gamma L_{\varepsilon}^{*}+\mu^{2}+\varepsilon \mu+\gamma \mu+\beta L_{\varepsilon}^{*} \mu-\beta H_{\varepsilon}^{*} \mu
\end{array}\right.
$$

are both positive if

$$
R_{0}=\frac{\beta \Lambda}{(\mu+\gamma)(\mu+\varepsilon)}>1
$$


From the Routh-Hurwitz theorem given in ${ }^{[26]}$, all roots of equation (5) have negative real parts. Consequently $E_{\varepsilon}{ }^{*}$ is locally asymptotically stable whenever $R_{0}>1$.

\section{Global stability of endemic equilibrium point for $\varepsilon>0$}

The following theorem discusses the global stability of the endemic equilibrium.

THEOREM 3.2. The endemic equilibrium $E_{\varepsilon}^{*}$ of the system (1) is globally asymptotically stable.

PROOF. Consider the following Lyapunov functional;

$$
W(t)=H_{\varepsilon}^{*} \phi\left(\frac{H(t)}{H_{\varepsilon}^{*}}\right)+L_{\varepsilon}^{*} \phi\left(\frac{L(t)}{L_{\varepsilon}^{*}}\right)
$$

Where $\phi(\mathrm{x})=\mathrm{x}-1-\ln (\mathrm{x}) \quad \mathrm{x} \in \mathbb{R}^{+}$. Obviously, $\phi: \mathbb{R}^{+} \rightarrow \mathbb{R}^{+}$ attains its global minimum; at $\mathrm{x}=1$ and $\phi(1)=0$.

To simplify the presentation, we shall use the following notation: $H=H(t)$ and $L=L(t)$.

$$
\begin{aligned}
& W(t)=H_{\varepsilon}^{*}\left(\frac{H(t)}{H_{\varepsilon}^{*}}-1-\ln \left(\frac{H(t)}{H_{\varepsilon}^{*}}\right)\right)+L_{\varepsilon}^{*}\left(\frac{L(t)}{L_{\varepsilon}^{*}}-1-\right. \\
& \left.\ln \left(\frac{L(t)}{L_{\varepsilon}^{*}}\right)\right) \\
& \begin{array}{c}
\frac{d W(t)}{d t}=\dot{H}\left(1-\frac{H_{\varepsilon}^{*}}{H}\right)+\dot{L}\left(1-\frac{L_{\varepsilon}^{*}}{L}\right) \\
=(\Lambda-\mu H-[\beta L+\varepsilon] H)\left(1-\frac{H_{\varepsilon}^{*}}{H}\right) \\
+\left(1-\frac{L_{\varepsilon}^{*}}{L}\right)([\beta L+\varepsilon] H \\
-(\gamma+\mu) L)
\end{array}
\end{aligned}
$$

Note that

$$
\Lambda=\mu H_{\varepsilon}^{*}+(\mu+\gamma)
$$

And

$$
\left(\beta L_{\varepsilon}^{*}+\varepsilon\right) H_{\varepsilon}^{*}=(\mu+\gamma) L_{\varepsilon}^{*}
$$

Hence,

$$
\begin{aligned}
\frac{d W(t)}{d t}=\left(1-\frac{H_{\varepsilon}^{*}}{H}\right) & \left(\mu H_{\varepsilon}^{*}+(\mu+\gamma) L_{\varepsilon}^{*}-\mu H\right. \\
- & (\beta L+\varepsilon) H) \\
& +\left(1-\frac{L_{\varepsilon}^{*}}{L}\right)((\beta L+\varepsilon) H \\
& -(\mu+\gamma) L) \\
\frac{d W(t)}{d t}=\mu\left(1-\frac{H_{\varepsilon}^{*}}{H}\right) & \left(H_{\varepsilon}^{*}-H\right)+(\mu+\gamma) L_{\varepsilon}^{*}\left(1-\frac{H_{\varepsilon}^{*}}{H}\right) \\
& -\left(1-\frac{H_{\varepsilon}^{*}}{H}\right)(\beta L+\varepsilon) H \\
& +\left(1-\frac{L_{\varepsilon}^{*}}{L}\right)(\beta L+\varepsilon) H \\
& -(\mu+\gamma) L\left(1-\frac{L_{\varepsilon}^{*}}{L}\right) \\
=\mu\left(1-\frac{H_{\varepsilon}^{*}}{H}\right) & \left(H_{\varepsilon}^{*}-H\right)-(\mu+\gamma) L_{\varepsilon}^{*}\left(\frac{H_{\varepsilon}^{*}}{H}\right. \\
& \left.+\frac{(\beta L+\varepsilon) H}{(\mu+\gamma) L}+\frac{(\mu+\gamma) L}{(\beta L+\varepsilon) H_{\varepsilon}^{*}}-3\right) \\
& +(\mu+\gamma) L_{\varepsilon}^{*}\left(-1-\frac{L}{L_{\varepsilon}^{*}}\right. \\
& +\frac{(\beta L+\varepsilon) H_{\varepsilon}^{*}}{(\mu+\gamma) L_{\varepsilon}^{*}}+\frac{(\mu+\gamma) L}{\left.(\beta L+\varepsilon) H_{\varepsilon}^{*}\right)} \\
=\mu\left(1-\frac{H_{\varepsilon}^{*}}{H}\right) & \left(H_{\varepsilon}^{*}-H\right) \\
& +(\mu+\gamma) L_{\varepsilon}^{*}\left(-1-\frac{L}{L_{\varepsilon}^{*}}\right. \\
& \left.+\frac{(\mu+\gamma) L}{H_{\varepsilon}^{*}(\beta L+\varepsilon)}+\frac{(\beta L+\varepsilon) H_{\varepsilon}^{*}}{(\mu+\gamma) L_{\varepsilon}^{*}}\right)-(\mu \\
& +\gamma) L_{\varepsilon}^{*}\left[\left(\phi\left(\frac{H_{\varepsilon}^{*}}{H}\right)+\ln \left(\frac{H_{\varepsilon}^{*}}{H}\right)\right.\right. \\
& +\phi\left(\frac{(\mu+\gamma) L}{H_{\varepsilon}^{*}(\beta L+\varepsilon)}\right) \\
& +\ln \left(\frac{(\mu+\gamma) L}{H_{\varepsilon}^{*}(\beta L+\varepsilon)}\right) \\
& +\phi\left(\frac{(\beta L+\varepsilon) H}{(\mu+\gamma) L}\right) \\
& \left.+\ln \left(\frac{(\beta L+\varepsilon) H}{(\mu+\gamma) L}\right)\right] \\
& \\
= &
\end{aligned}
$$

Then, we obtain the following equation:

$$
\begin{aligned}
\frac{d W(t)}{d t}=\mu\left(H_{\varepsilon}^{*}-\right. & H)\left(1-\frac{H_{\varepsilon}^{*}}{H}\right) \\
+ & (\mu+\gamma) L_{\varepsilon}^{*}\left(-1-\frac{L}{L_{\varepsilon}^{*}}\right. \\
& \left.+\frac{(\mu+\gamma) L}{H_{\varepsilon}^{*}(\beta L+\varepsilon)}+\frac{(\beta L+\varepsilon) H_{\varepsilon}^{*}}{(\mu+\gamma) L_{\varepsilon}^{*}}\right) \\
& -(\mu+\gamma) L_{\varepsilon}^{*}\left(\phi\left(\frac{H_{\varepsilon}^{*}}{H}\right)\right. \\
& +\phi\left(\frac{(\mu+\gamma) L}{H_{\varepsilon}^{*}(\beta L+\varepsilon)}\right) \\
& \left.+\phi\left(\frac{(\beta L+\varepsilon) H}{(\mu+\gamma) L}\right)\right]
\end{aligned}
$$

From equation (6), we have: 


$$
\begin{aligned}
\frac{d W(t)}{d t}=\mu\left(H_{\varepsilon}^{*}-\right. & H)\left(1-\frac{H_{\varepsilon}^{*}}{H}\right) \\
+ & (\mu \\
& +\gamma) L_{\varepsilon}^{*}\left(\frac{-\varepsilon \beta\left(L-L_{\varepsilon}^{*}\right)^{2}}{L_{\varepsilon}^{*}(\beta L+\varepsilon)\left(\beta L_{\varepsilon}^{*}+\varepsilon\right)}\right) \\
& -(\mu+\gamma) L_{\varepsilon}^{*}\left(\phi\left(\frac{H_{\varepsilon}^{*}}{H}\right)\right. \\
& +\phi\left(\frac{(\mu+\gamma) L}{H_{\varepsilon}^{*}(\beta L+\varepsilon)}\right) \\
& \left.+\phi\left(\frac{(\beta L+\varepsilon) H}{(\mu+\gamma) L}\right)\right]
\end{aligned}
$$

Since,

$$
\left\{\begin{array}{c}
-\frac{\mu}{H}\left(H-H_{\varepsilon}^{*}\right)^{2} \leq 0 \\
\frac{-\varepsilon \beta\left(L-L_{\varepsilon}^{*}\right)^{2}}{L_{\varepsilon}^{*}(\beta L+\varepsilon)\left(\beta L_{\varepsilon}^{*}+\varepsilon\right)} \leq 0 \\
-(\mu+\gamma) L_{\varepsilon}^{*}\left[\phi\left(\frac{H_{\varepsilon}^{*}}{H}\right)+\phi\left(\frac{(\mu+\gamma) L}{H_{\varepsilon}^{*}(\beta L+\varepsilon)}\right)+\phi\left(\frac{H(\beta L+\varepsilon)}{(\mu+\gamma) L}\right)\right] \leq 0
\end{array}\right.
$$

We have

$$
\frac{d W(t)}{d t} \leq 0
$$

Thus $E_{\varepsilon}^{*}$ is stable, and

$$
\frac{d W(t)}{d t}=0
$$

if and only if $\mathrm{H}=\mathrm{H}_{\varepsilon}{ }^{*}$ and $\mathrm{L}=\mathrm{L}^{*}$.

From LaSalle invariance principle ${ }^{[27]}$, we conclude that $E_{\varepsilon}^{*}$ is globally asymptotically stable. Since $\phi(\mathrm{x}) \geq$ 0 , we have

$$
\frac{\mathrm{dW}(\mathrm{t})}{\mathrm{dt}} \leq 0
$$

\section{Stability analysis for special case $\varepsilon=0$}

$$
\begin{aligned}
& \frac{\mathrm{dH}}{\mathrm{dt}}=\Lambda-\mu \mathrm{H}-\beta \mathrm{LH}, \\
& \frac{\mathrm{dL}}{\mathrm{dt}}=\beta \mathrm{LH}-(\mu+\gamma) \mathrm{L}, \\
& \frac{\mathrm{dR}}{\mathrm{dt}}=\gamma \mathrm{L}-\mu \mathrm{R} .
\end{aligned}
$$

Notice that the system (8) has a basic reproductive number

$$
\mathrm{R}_{0}=\frac{\beta \Lambda}{\mu(\mu+\gamma)}
$$

$R_{0}$ represents the average number of secondary infections caused by an infective individual introduced into a group of susceptible. By equalizing to zero the rights members of the system (8), we find two equilibrium points that exists for above model:

1. Disease-free equilibrium point $E_{f}$

$$
\mathrm{E}_{\mathrm{f}}=\left(\frac{\Lambda}{\mu}, 0,0\right)
$$

2. Endemic equilibrium point $E^{*}=\left(H^{*}, L^{*}, R^{*}\right)$ where,

$$
\mathrm{H}^{*}=\frac{(\mu+\gamma)}{\beta}
$$

$$
\begin{aligned}
& \mathrm{L}^{*}=\mu \frac{\left(\beta \frac{\Lambda}{\mu}-\mu-\gamma\right)}{\beta(\mu+\gamma)} \\
& \mathrm{R}^{*}=\gamma \frac{\left(\beta \frac{\Lambda}{\mu}-\mu-\gamma\right)}{\beta(\mu+\gamma)}
\end{aligned}
$$

The endemic equilibrium point exist only when

$$
\beta \frac{\Lambda}{\mu}>\mu+\gamma
$$

i.e the infection rate must be greater than the death rate of the infected individuals or $R_{0}>1$.

\section{Local stability of equilibria}

The following theorems discuss the local stability of the equilibrium point.

\section{THEOREM 3.3.}

1. If $\mathrm{R}_{0}<1$, then the disease-free equilibrium, $E_{f}$ is locally asymptotically stable.

2. If $R_{0}>1, E_{f}$ is unstable.

PROOF. The Jacobian matrix evaluated in the disease-free equilibrium 


$$
\mathrm{E}_{\mathrm{f}}=\left(\frac{\Lambda}{\mu}, 0,0\right)
$$

is given by

$$
J\left(E_{f}\right)=\left(\begin{array}{ccc}
-\mu & -\beta \frac{\Lambda}{\mu} & 0 \\
0 & \beta \frac{\Lambda}{\mu}-(\mu+\gamma) & 0 \\
0 & \gamma & -\mu
\end{array}\right)
$$

Whose eigenvalues are $\lambda_{1}=-\mu<0$ and

$$
\left(\beta \frac{\Lambda}{\mu}-\gamma-\mu\right)<0
$$

then $\mathrm{R}_{0}<1$ and therefore $\mathrm{E}_{\mathrm{f}}$ is locally asymptotically stable.

The disease-free equilibrium point is unstable if

$$
\left(\beta \frac{\Lambda}{\mu}-\gamma-\mu\right)>0
$$

which translate into $R_{0}>1$.

Now, we focus on local stability of the endemic infection equilibrium $E^{*}$.

\section{THEOREM 3.4.}

1. If $R_{0}>1, E^{*}$ is locally asymptotically stable.

2. If $R_{0}<1$, then the endemic equilibrium $E^{*}$ does not exist.

PROOF. By substituting the endemic equilibrium $E^{*}=\left(H^{*}, L^{*}, R^{*}\right)$ in the Jacobien matrix of the system (8)

$$
J\left(E^{*}\right)=\left(\begin{array}{ccc}
-\mu\left(\frac{\beta \frac{\Lambda}{\mu}-\gamma-\mu}{\gamma+\mu}+1\right) & -(\gamma+\mu) & 0 \\
\mu\left(\frac{\beta \frac{\Lambda}{\mu}-\gamma-\mu}{\gamma+\mu}\right) & 0 & 0 \\
0 & \gamma & -\mu
\end{array}\right)
$$

The characteristic equation of the endemic equilibrium point is given by

$$
(-\mu-\lambda)\left(\lambda^{2}+\frac{\beta \Lambda \lambda}{\mu+\gamma}+\mu\left(\beta \frac{\Lambda}{\mu}-\gamma-\mu\right)\right)=0 \text { (9) }
$$

Clearly when $\mathrm{R}_{0}>1$, both

$$
\frac{\beta \Lambda \lambda}{\mu+\gamma}>0
$$

And

$$
\mu\left(\beta \frac{\Lambda}{\mu}-\gamma-\mu\right)>0
$$

then all roots of the characteristic equation have negative real parts. Consequently $\mathrm{E}^{*}$ is locally asymptotically stable.

\section{Global stability of equilibria}

In this section, we establish the global stability of the equilibria. Firstly, we have the following

THEOREM 3.5. The disease-free equilibrium $E_{f}$ is globally asymptotically stable when $R_{0}<1$.

PROOF. Consider the following Lyapunov functional

$$
V_{1}(t)=H_{f}\left(\frac{H}{H_{f}}\right)+L
$$

Where

$$
H_{f}=\frac{1}{\mu}
$$

and calculating the time derivative of $\mathrm{V}_{1}(\mathrm{t})$ along the positive solution of system (8), we get;

$$
\begin{aligned}
V_{1}(t) & =\dot{H}\left(1-\frac{H_{f}}{H}\right)+\dot{L} \\
& =\left(1-\frac{H_{f}}{H}\right)(\Lambda-\mu H-\beta H L)+\beta H L-(\mu+\gamma) L \\
& =\left(1-\frac{H_{f}}{H}\right)(\Lambda-\mu H)+\beta H_{f} \mathrm{~L}-(\mu+\gamma) L
\end{aligned}
$$


Where $\Lambda=\mu H_{f}$, we have;

$$
\frac{d V_{1}}{d t}(t)=\mu\left(1-\frac{H_{f}}{H}\right)\left(H_{f}-H\right)+\left(R_{0}-1\right) L
$$

Since $R_{0} \leq 1$, we have

$$
\frac{d V_{1}}{d t}(t) \leq 0
$$

Thus, the disease-free equilibrium $E_{f}$ is stable, and

$$
\frac{d V_{1}}{d t}(t)=0
$$

if and only if $H=H_{f}$ and $L\left(R_{0}-1\right)=0$. We discuss two cases:

, If $\mathrm{R}_{0}<1$, then $\mathrm{L}=0$.

, If $\mathrm{R}_{\mathrm{o}}=1$. From $H=H_{f}$ and the first equation of the system (8), we have

$$
\frac{d H}{d t}=\frac{d H_{f}}{d t}=\Lambda-\mu H_{f}-\beta H_{f} L=0
$$

Then $\beta H_{f} L=0$, Hence $L=0$.

From LaSalle invariance principle ${ }^{[27]}$, we conclude that $E_{f}$ is globally asymptotically stable.

Note that the disease-free equilibrium $E_{f}$ is unstable when $R_{0}>1$.

Now, we establish a set of conditions which are sufficient for the global stability of the endemic equilibrium $E^{*}$.

THEOREM 3.6. The endemic equilibrium $\mathrm{E}^{*}$ of the system (8) is globally asymptotically stable.

PROOF. See the proof of theorem 3.2, and we replace $\varepsilon$ by zero, we conclude that $\mathrm{E}^{*}$ is globally asymptotically stable.

\section{NUMERICAL SIMULATIONS}

In this section, we show the numerical simulations and the graphs of system (1) to illustrate the different result obtained for each of the two cases $\varepsilon>0$ and $\varepsilon=$ 0 previously analyzed.

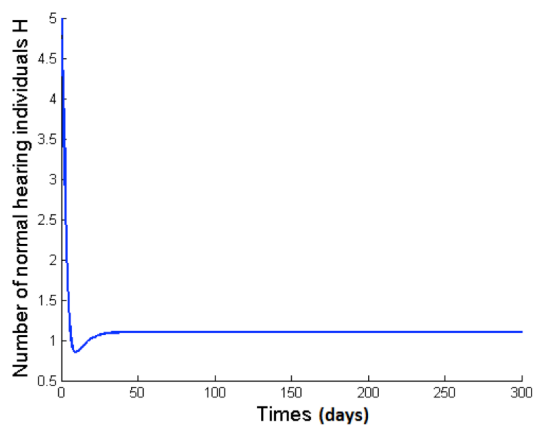

a)

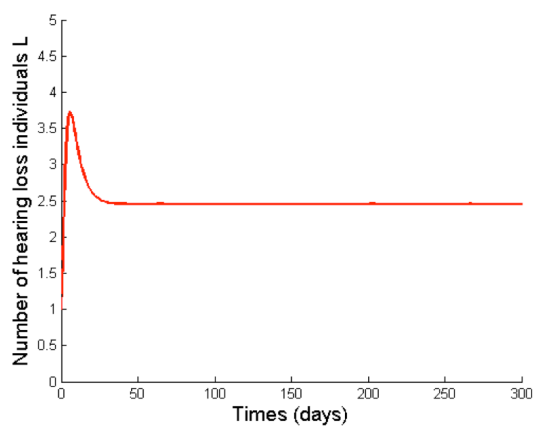

b)

\section{FIGURE 1. Normal hearing (a) and loss of hearing (b) individuals as function of time in the case of $\varepsilon=0,0025$ and $R_{0}>1$.}

We choose the following data set of system (1) as follows: $\Lambda=0.5, \mu=0.1$ and $\gamma=1 / 17$. By using the values of $\Lambda, \mu, \gamma$ and $\varepsilon=0.0025>0{ }^{[28]}$, we find $\beta=0.1433$ for $R_{0}>$ 1 already defined for mumps disease ${ }^{[29]}$. Therefore, according to Theorem 3.2, the system (1) has a unique endemic equilibrium which is globally asymptotically stable. So, the solution of system (1) is persistent in population and converge to $E_{\varepsilon}^{*}=(1,1008 ; 2,4450)$, this results are illustrated in Figure 1. Now, we choose $\varepsilon=0$ which means the hearing loss result only from mumps diseases. So, the system (1) has a disease free equilibrium $E_{f}=(5,0)$ which is globally asymptotically stable when $R_{0}<1$, then the disease dies out. Numerical simulations illustrated our results (see Figure 2). For $R_{0}=$ $4.4>1$, the endemic $E^{*}$ is globally asymptotically sta- 


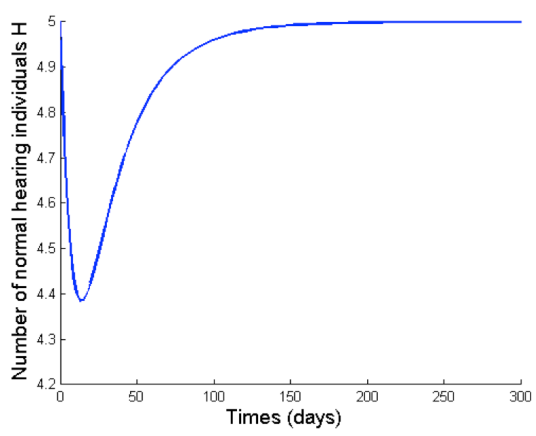

a)

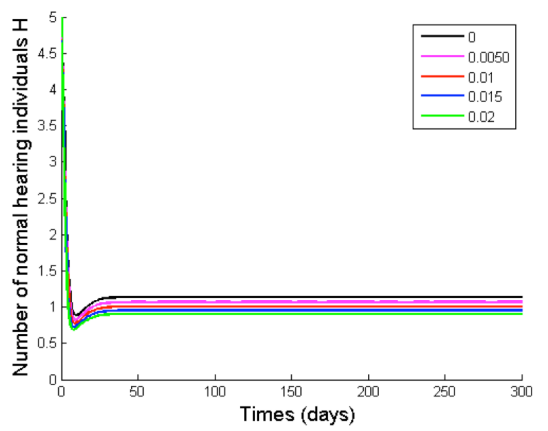

a)
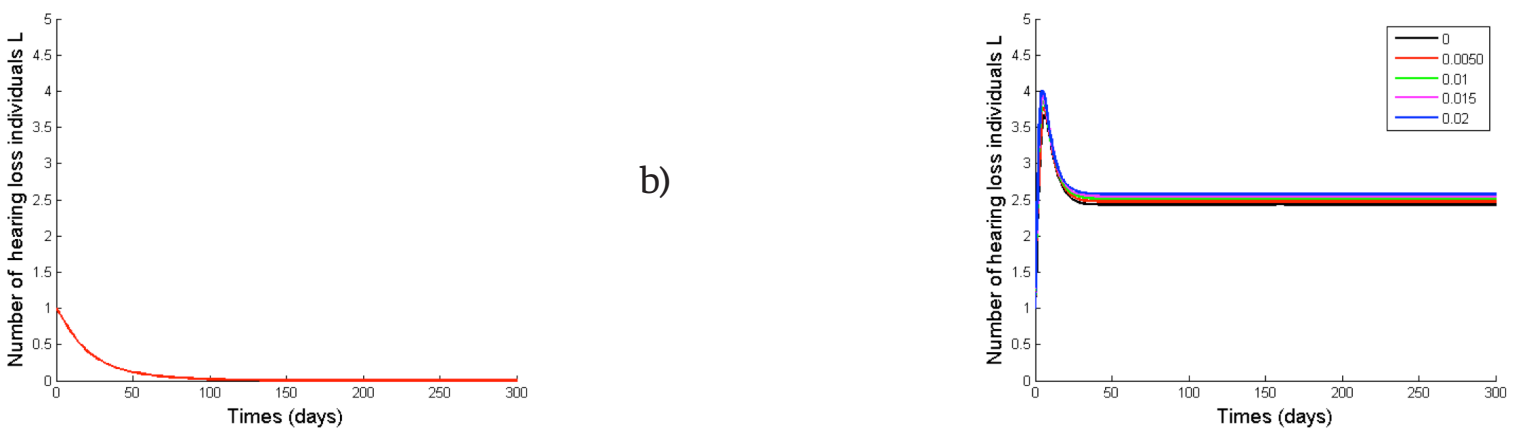

b)

FIGURE 2. Normal hearing (a) and loss of hearing (b) individuals as function of time in the case of $\varepsilon=0$ and $R_{0}<1$.

b)
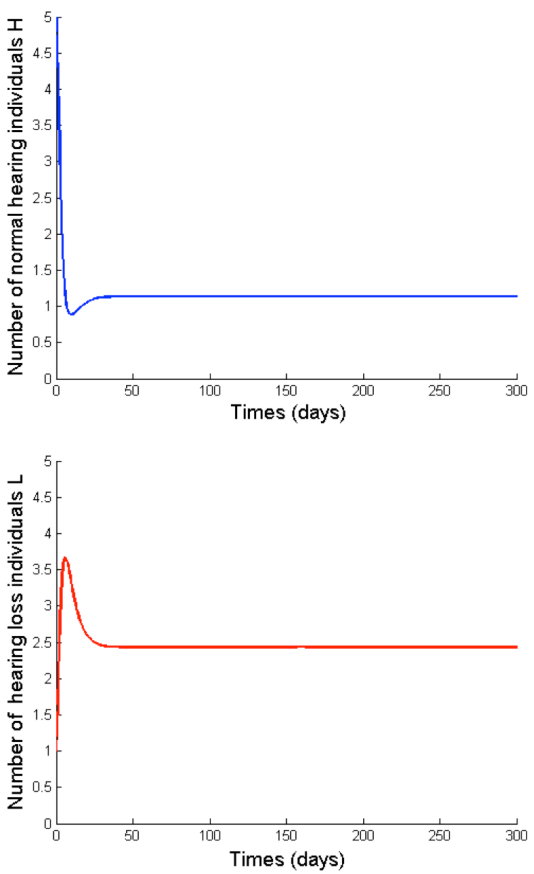

FIGURE 3. Normal hearing (a) and loss of hearing (b) individuals as function of time in the case of $\varepsilon=0$ and $R_{0}>1$. a)

b)

\section{CONCLUSIONS}

In this paper, we have presented a mathematical model of hearing loss based on a nonlinear system of differential equations. We analysis the hearing loss resulting from two factors, the first factor is contagious due to Mumps disease and the second is social caused by exposure to noise. By analysis the model, we have proved the existence, positivity and the boundedness of solutions of the problem, which implies that the model is well posed. We have shown in the case of $\varepsilon=0$ that the disease free equilibrium is globally asymptotically stable if the basic reproductive number $R_{0}<1$ and 
the endemic point is globally asymptotically stable when $R_{0}>1$. In the case of hearing loss with the both factors mumps and noise $\varepsilon>0$ the system has a unique endemic point exists and is globally asymptotically stable, which means that the disease persists in the population. In addition, the simulation of this model provides that the number of individuals with hearing loss increase when we introduce the risk factor noise. 


\section{REFERENCES}

[1] WHO, Deafness and hearing loss, Fact sheet Updated February 2017 Available at: $h t t p: / / w w w . w h o . i n t / m e d i a c e n t r e / f a c t s h e e t s / f s 300 / e n$

[2] K. Ravindra, J. Best, E. MacMahon, Mumps and the UK Epidemic 2005, BMJ 330 (7500) (2005) 1132-1135.

[3] B. E. Cohen, A. Durstenfeld, P. C. Roehm, Viral causes of hearing loss: a review for hearing health professionals, Trends in hearing 18 (2014) 1-17.

[4] S. Morita, K. Fujiwara, A. Fukuda, The clinical features and prognosis of mumps-associated hearing loss: a retrospective, multi-institutional investigation in Japan, Acta oto-laryngologica, 137 (2017) 1-4.

[5] S. Hammer, K. Swinburn, L. Neitzel, Environmental noise pollution in the United States: developing an effective public health response, Environmental health perspectives, 122 (2014) 115-119.

[6] M. Basner, W. Babisch, A. Davis, M. Brink, C. Clark, S. Janssen, S. Stansfeld, Auditory and non-auditory effects of noise on health, The Lancet, 383 (9925) (2014)1325-1332.

[7] FZ. Aboulkhouatem, F. Kouilily, M. EL Khasmi, N. Achtaich, N. Yousfi, The Active Model: The Effect of Stiffness on the Maximum Amplitude Displacement of the Basilar Membrane, British Journal of British Journal of Mathematics \& Computer Science, 20 (BJMCS.30856) (2017) 1-11.

[8] S. Lee, T. Koike, Simulation of the Basilar Membrane Vibration of Endolymphatic hydrops, Procedia IUTAM, 24 (2017) 64-71.

[9] S. T Neely, Finite difference solution of a two-dimensional mathematical model of the cochlea, J. Acoust. Soc. Amer., 69 (5) (1981) 1386-1393.

[10] H. M. Jiménez, Computational Model of the Cochlea using Resonace Analysis, Rev. Mex. Ing. Biomédica, 33(2) (2012) 77-86.

[11] F. Kouilily, FZ. Aboulkhouatem, N. Yousfi, N. Achtaich, Predicting the Effect of Physical Parameters on the Amplitude of the Passive Cochlear Model, Rev. Mex. Ing. Biomédica 39 (1) (2018) 105-112.

[12] J. Graunt, Natural and political observations made upon the bills of mortality, Roycroft and Dicas: London (1662).

[13] W.O. Kermack, A.G. Mckendric, Contribution to the mathematical theory of epidemics, Proceedings of the Royal Society of London A, 115 (1927) 700-721.

[14] X. Wang, K. Hattaf , H. Huo, H. Xiang, Stability analysis of a delayed social epidemic model with general contact rate and its optimal control, Journal of Industrial \& Management Optimization, 12 (4) $1267-1285$.

[15] K. Hattaf, N. Yousfi, Mathematical model of the influenza A (H1N1) infection, Advanced Studies in Biology, 1 (8) (2009) 383-390.
[16] K. Hattaf, A.Lashari, Y. Louartassi, N. Yousfi, A delayed SIR epidemic model with a general incidence rate, Electronic Journal of Qualitative Theory of Differential Equations, (3) (2013) 1-9.

[17] A. A. Lashari, G. Zaman, Global dynamics of vector-borne diseases with horizontal transmission in host population, Computers \& Mathematics with Applications, 61(4) (2011) 745-754.

[18] R. Xu, Z. Ma, Global stability of a SIR epidemic model with nonlinear incidence rate and time delay, Nonlinear Analysis: Real World Applications, 10(5) (2009) 3175-3189.

[19] K. Hattaf, N. Yousfi, A. Tridane, Stability analysis of a virus dynamics model with general incidence rate and two delays, Applied Mathematics and Computation, 221 (2013) 514-521.

[20] S. M. Guo, X. Z. Li, X. Y. Song, Stability of an age-structured SEIS epidemic model with infectivity in incubative period, International Journal of Biomathematics, 3(03) (2010) 299-312.

[21] L. M. Cai, X. Z. Li, Global analysis of a vector-host epidemic model with nonlinear incidences, Applied Mathematics and Computation, 217(7) (2010) 3531-3541.

[22] F. Li, N. K. Yip, Long time behavior of some epidemic models, Discrete and continuous dynamical systems series B, 16 (2011) 867-881.

[23] S. J. Chang, C. J. Chen, C. H. Lien, F. C. Sung, Hearing loss in workers exposed to toluene and noise, Environmental Health Perspectives, 114(8) (2006) 1283-1286.

[24] D. Henderson, E. C. Bielefeld, , K. C. Harris, B. H. Hu, The role of oxidative stress in noise-induced hearing loss, Ear and hearing, 27(1) (2006) 1-19.

[25] N. Slepecky, Overview of mechanical damage to the inner ear: noise as a tool to probe cochlear function, Hearing research, 22(1-3) (1986) 307-321.

[26] I. S. Gra dshteynet, I. M. Ryzhik, Routh-hurwitz theorem, Tables of integrals, series, and products, (2000).

[27] J. P. LaSalle The stability of dynamical systems, Siam 25 (1976).

[28] I. I. Bogoch, R. A. House, I. Kudla, Perceptions about hearing protection and noise-induced hearing loss of attendees of rock concerts, Canadian Journal of Public Health/Revue Canadienne de Santée Publique, 96 (1) 69-72.

[29] W. J. Edmunds, N. J. Gay, M. Kretzschmar, R. G. Pebod, H. Wachmann, The pre-vaccination epidemiology of measles, mumps and rubella in Europe: implications for modeling studies, Epidemiology \& Infection, 125 (3) (2000) 635-650. 